\title{
Cartografía elaborada con un método fotogramétrico simple versus un método digital (Softcopy-Fotogrametría)
}

\author{
Cartography undertaken using simple photogrammetry as compared with \\ digital methods
}

VICTOR SANDOVAL

Facultad de Ciencias Forestales, Universidad Austral de Chile. E-mail: vsandova@uach.cl

\begin{abstract}
SUMMARY
The magnitude of error made in plotting the spatial position of objects mapped using simple photogrammetry methods was examined for the 61,000 ha area between the central valley and the frontal mountains of the Eighth Region which is the subject of this paper. Comparative cartography for the area was obtained from map drawn digitally using the Softcopy-Photogrammetry concept. It was concluded from these test results that the highland area on the existing cartography for the Catastro project had an absolute error close to $5 \%$ in spatial positioning derived from polygonal surveys of the surface. One conclusion was that the real surface error, excluding compensation and underestimation, was close to $12 \%$.
\end{abstract}

Key words: GIS, remote sensing, photogrammetry.

\section{RESUMEN}

Se evaluó el error de la cartografía de uso actual del suelo, elaborada con métodos fotogramétricos simples, según la metodología utilizada en el Proyecto Catastro y Evaluación de los Recursos Vegetacionales de Chile. El área de prueba se ubicó entre el valle central y la precordillera costera de la VIII Región, sobre una superficie de 61.000 ha. El método de referencia correspondió a cartografía elaborada con procedimientos cartográficos digitales o Softcopy-Fotogrametría. Los resultados obtenidos permiten concluir que la documentación cartográfica elaborada con métodos fotogramétricos sencillos tiene un error absoluto cercano al 5\% en la posición espacial de los polígonos y un error real en la estimación de la superficie de aproximadamente un $12 \%$ en el área de prueba. En este estudio no se evaluaron los costos de producción de cartografía digital, por cuanto éstos radican principalmente en el valor específico del programa de restitución digital que se utilice.

Palabras claves: SIG, sensores remotos, fotogrametría digital.

\section{INTRODUCCION}

La cartografía oficial de uso del suelo del país, levantada durante el período 1994 a 1997, en el marco del proyecto "Catastro y evaluación de los recursos vegetacionales nativos de Chile", fue elaborada con métodos fotogramétricos sencillos (1). Las principales fases cartográficas que utiliza esta metodología corresponden a la fotointerpretación del uso del suelo, control de campo y transferencia a una base cartográfica, mediante el Sketch- master vertical de la firma Zeiss (2). La cartografía producida con ese método tiene algunas deficiencias en la exactitud espacial de los objetos mapeados (3). Este error se produce principalmente por el desplazamiento radial existente en las áreas bordes de los fotogramas verticales y por las características de los puntos o líneas de control seleccionadas (4).

Con el objeto de determinar la magnitud de los errores cartográficos descritos, se evaluó la carta IGM-F101, elaborada durante el proyecto Catas- 
BOSQUE 25(1): 87-94, 2004

Cartografía elaborada con un método fotogramétrico simple versus un método digital (Softcopy-Fotogrametría)

tro, respecto a un mapa de referencia elaborado con técnicas de restitución digital, perteneciente a la línea Softcopy-Fotogrametría. El área específica corresponde a un sector precordillerano de la VIII Región. La carta de referencia se confeccionó específicamente con el software fotogramétrico Softplotter versión 1.7.

\section{SOFTCOPY-FOTOGRAMETRIA}

El proceso de traspaso de información desde fotografías aéreas a una base cartográfica, mediante el instrumento "Sketchmaster", es aún ampliamente utilizado en Chile. Este método ofrece algunas ventajas cuando se elaboran mapas de extensas superficies con fotografías aéreas verticales de escala media (1:15.000-1:40.000) (2). Estas ventajas radican principalmente en los siguientes aspectos:

a) Rapidez en la transferencia de la información a la carta base.

b) Bajos costos de instrumental.

c) Bajo tiempo de preparación y experiencia de operadores.

La principal desventaja de la cartografía producida con este método corresponde a la falta de exactitud en la posición espacial de superficies previamente fotointerpretadas (2).

La cartografía elaborada bajo el concepto de Softcopy-Fotogrametría emplea programas que posibilitan, en forma automatizada, la orientación relativa y absoluta de los fotogramas, la fotointerpretación y medición de objetos en forma simultánea, mediante el principio marca flotante o paralaje $\mathrm{x}(3)$.

La calidad y exactitud de la cartografía elaborada con esta metodología digital depende en general de la resolución y calidad del material fotográfico utilizado, del tipo de Scanner y de la visibilidad de los puntos de control empleados (5).

Entre las principales ventajas y desventajas de un sistema fotogramétrico digital como el Softplotter utilizado en este estudio, se pueden citar las siguientes:

Ventajas

a) Transportabilidad de la información.

b) Orientación interna y relativa es automática. c) Posibilidad que muchos usuarios pueden usar simultáneamente los estéreo modelos.

d) Automatización en el ingreso de atributos.

e) Medición y restitución son procesos automatizados.

f) Software para mediciones fotogramétricas. Ortorrectificación y sistemas de información geográficos son integrados y disponibles en una misma plataforma.

\section{Desventajas}

a) Fotografías aéreas deben ser "scanneadas".

b) Alta necesidad de espacio para almacenar información digital.

c) Disminución de la calidad en las fotografías aéreas escaneadas respecto a las fotos originales.

\section{MATERIAL Y METODO}

En este estudio se considera como Método 1 la cartografía elaborada durante el Proyecto Catastro. La cartografía digital de referencia o Método 2 corresponde a la confeccionada con el programa Softplotter versión 1.7. Para la elaboración de esta se emplearon diapositivas color escala 1:115.000, "scanneadas" a una resolución de $30 \mu \mathrm{m}$, lo que determina una resolución 3,45 metros en terreno (cuadro 1).

En el cuadro 1 se presenta una descripción técnica detallada del material fotográfico utilizado en ambos métodos de producción cartográfica.

La cartografía referencial (Método 2), elaborada mediante el uso de esta moderna tecnología, se realizó empleando las funcionalidades pertenecientes al Integred Photogrammetric System (IPS) del Softplotter 1.7. Previo a las operaciones de importación de información de la imagen fotográfica, se definieron especificaciones del proyecto, que incluyen el datum de referencia, altitud promedio del terreno, porcentaje de traslapo lateral y frontal entre diapositivas, tipo de proyección cartográfica a utilizar e información de calibración de la cámara aérea utilizada.

El diagrama de flujo (figura 1) indica la secuencia de actividades realizadas para la elaboración de la cartografía referencial de este estudio. Los principales módulos operativos utilizados en esta fase y su funcionalidad son los siguientes: 


\section{CUADRO 1}

Descripción técnica del material fotográfico utilizado. Technical description of photographic materials used.

\begin{tabular}{|l|l|l|}
\hline Descripción de foto aérea & Método 1 & Método 2 \\
\hline Nombre de vuelo & FONDEF & CONAF \\
Fecha & Enero, 1994 & Marzo, 1998 \\
Hora & 11 AM (Hora local) & 12 AM (Hora local) \\
Cámara & Wild 1053 & Wild RC-Leica 8.8 Sag II \\
Distancia focal & 153,3 & 88,09 \\
Tipo de film & Pancromático B.N & Color \\
Escala promedio & $1: 20.000$ & $1: 115.000$ \\
Altura de vuelo & 3.000 ms.n.m. & 10.000 ms.n.m. \\
Dirección línea de vuelo & N->S & N->S \\
Traslapo frontal & $60 \%$ & $55 \%$ \\
Traslapo lateral & $20 \%$ & $25 \%$ \\
Número de fotos & 130 & 4 \\
\hline
\end{tabular}

a) Importación, orientación de la imagen y triangulación (Block-tool): Corresponde a la definición de parámetros de la cámara aérea empleada, importación de la imagen fotográfica digital, ingreso y medición de los puntos de control, orientación de la imagen bajo el principio de colinealidad y triangulación.

b) Generación de modelos estereoscópicos (Stereo Tool): Se refiere a la rectificación de la imagen triangulada bajo una orientación "epipolar", de tal forma que el paralaje en la línea de vuelo (y) es eliminado, y el paralaje (x) es interpretado como diferencia de elevación, se obtiene un modelo estereoscópico del área.

c) Modelo de elevación de terreno (MET) (Surface Tool): Corresponde a una descripción numérica tridimensional de la superficie del área, mediante el uso de las coordenadas (X, Y, Z). En este estudio se elaboró un MET, empleando la técnica "Triangulated Irregular Network" (TIN), función integrada al Softplotter.

d) Ortoreectificación (Ortho Tool): Es el proceso corrección diferencial de la proyección central de la fotografía aérea, para trasformarla en una proyección ortogonal.

e) Mosaicos (Mosaic Tool): Corresponde a la unión de las ortofotos, utilizando el área de traslapo entre ellas (área de menor desplaza- miento por efecto del relieve en cada ortofoto). La función "Compute Histogram Matching" permite transformar cada componente de la imagen en el mosaico, minimizando el contraste entre las diferentes ortofotos.

e) Fotointerpretación del uso del suelo (KDMSTool): Para evaluar la calidad de la cartografía elaborada el año 1994, se delinearon e interpretaron sobre el ortofotomosaico y bajo visión estereoscópica las distintas clases de uso de la tierra definidas por la cartografía del Proyecto Catastro. En este proceso se utilizó la herramienta KDMS incluida en el Softplotter. Esta herramienta permite delinear los elementos del paisaje bajo visión 3D y en formato vectorial. Las unidades o polígonos delineados con el Softplotter (Método 2) constituyeron la base de referencia para juzgar la calidad de la cartografía del Proyecto Catastro en la zona de estudio.

Evaluación de la calidad de la cartografía elaborada con el Método 1. La calidad de la cartografía geométrica se evaluó, comparando la posición espacial y límites de los elementos mapeados con el Método 1, respecto a la cartografía digital elaborada con el Método 2. Las expresiones de error definidas para este proceso fueron las siguientes: 
BOSQUE 25(1): 87-94, 2004

Cartografía elaborada con un método fotogramétrico simple versus un método digital (Softcopy-Fotogrametría)

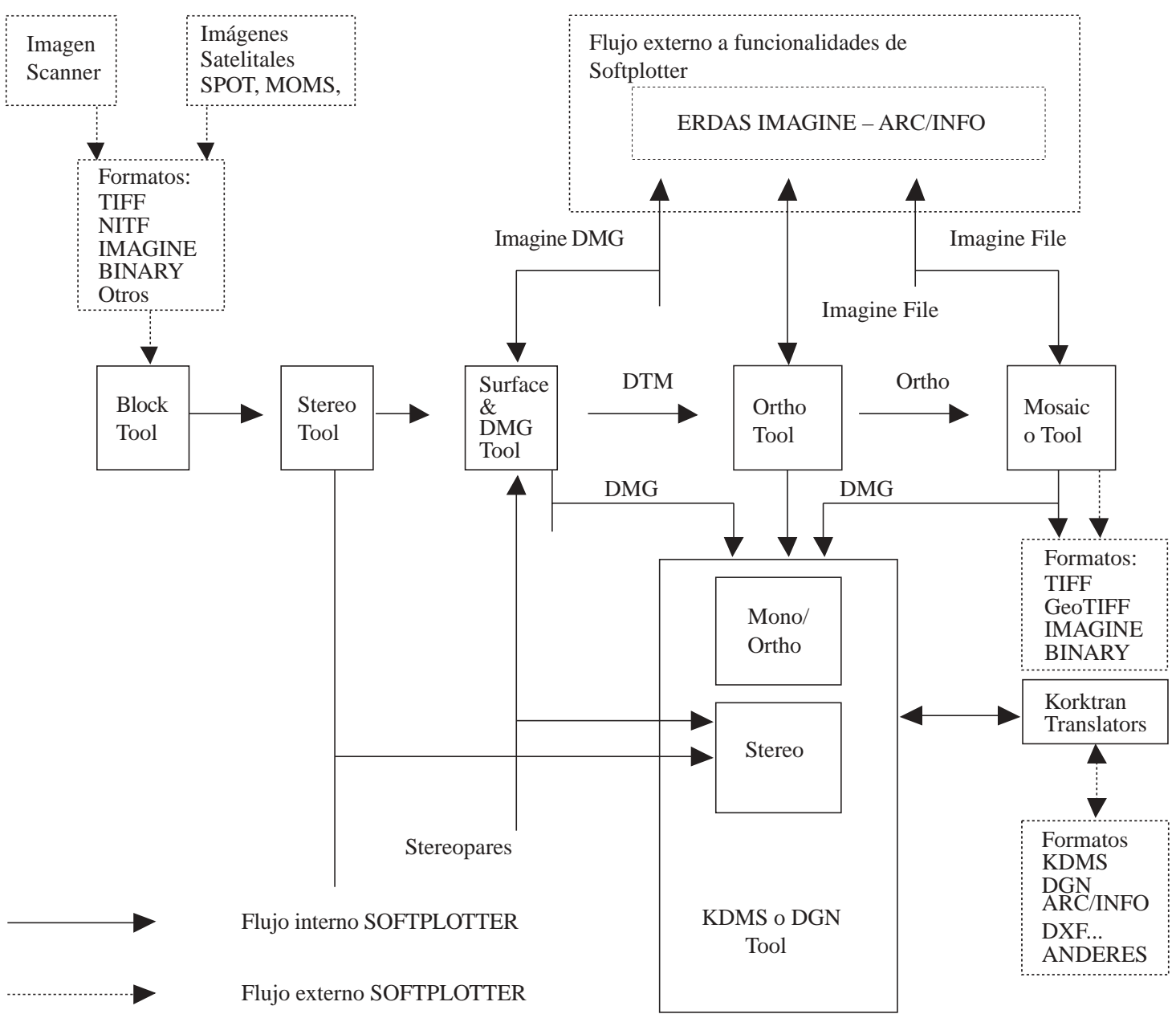

Figura 1. Diagrama de Flujo y Funcionalidades del SOFTPLOTTER.

Flow diagram and functionalities of the SOFTPLOTTER.

\section{- Errores de Posición}

a) Error de coordenadas $\mathrm{x}$ e y $\left(\mathrm{d}_{\mathrm{x}} \mathrm{y} \mathrm{d}_{\mathrm{y}}\right)$ : Representa el error de localización espacial de un punto p en la cartografía elaborada con el (Método 1), respecto a la posición real de $\mathrm{p}$ en terreno (Método 2). La cuantificación de este error se realizó midiendo una muestra de 150 puntos, distribuidos sistemáticamente en toda la superficie de estudio. Desde cada punto de muestreo se midieron las coordenadas $\mathrm{x}$ e y de un punto $\mathrm{p}$ inequívocamente identificable en ambas cartas (Ej. cruces de caminos, intersección de dos clases de uso del suelo, etc.). La magnitud se evaluó de acuerdo a la siguiente expresión: $\mathrm{d}_{\mathrm{x}}=\mathrm{x}_{\text {método 1 }}-\mathrm{x}_{\text {método } 2} \mathrm{y} \mathrm{d}_{\mathrm{y}}=\mathrm{y}_{\text {método 1 }}-\mathrm{y}_{\text {método } 2}[1]$

b) Error de Posición $d_{p}$ : Se refiere al error de posición y orientación espacial de un punto $\mathrm{p}$ en la cartografía elaborada con el Método 1 , respecto a su posición real en terreno. La información para cuantificar $\mathrm{d}_{\mathrm{p}}$ se obtuvo a partir de los 150 puntos de muestreo utilizados en la cuantificación de los errores en las coordenadas $\mathrm{x}$ e $\mathrm{y}$.

El error de posición $d_{p}$ se evaluó con la conocida fórmula:

$$
d_{p}=\sqrt{d_{x}^{2}+d_{y}^{2}}
$$


BOSQUE 25(1): 87-94, 2004

Cartografía elaborada con un método fotogramétrico simple versus un método digital (Softcopy-Fotogrametría)

\section{- Error de Superficie}

Corresponde a la diferencia $\mathrm{d}_{\mathrm{si}}$ de superficie entre la cartografía elaborada entre ambos métodos. La determinación de este error se obtuvo midiendo una muestra de 42 polígonos, cuyas superficies variaron entre 17 y 1.637 ha. La cuantificación de este error se evaluó considerando también las diferencias de sobre o subestimación existentes.

El error de superficies entre ambas cartas se define como:

$$
\mathrm{d}_{\mathrm{si}}=\mathrm{Si}_{\text {Método } 1}-\mathrm{Si}_{\text {Método } 2}
$$

Donde:

$\mathrm{d}_{\mathrm{si}} \quad$ : diferencia absoluta entre las superficies $\mathrm{S}_{\text {Método 1 }}-\mathrm{S}_{\text {Método 2 del polígono i. }}$

$\mathrm{Si}_{\text {Método 1 }}$ : Superficie del polígono i en la carta elaborado con el Método 1.

$\mathrm{Si}_{\text {Método } 2}$ : Superficie del polígono i de carta elaborado con el Método 2.

El error de subestimación o de sobrestimación, es resultante del signo de la diferencia de superficies. En este caso se consideró error de subestimación cuando $\mathrm{d}_{\mathrm{si}}=\mathrm{Si}_{\text {Método } 1}-\mathrm{Si}_{\text {Método } 2}$ es positivo $\mathrm{y}$, en caso contrario, se considera como sobrestimación o diferencia con signo negativo.

\section{RESULTADOS Y DISCUSION}

La calidad y ordenamiento de la información cartográfica y alfanumérica juega un importante rol en cualquier proyecto de dimensionamiento o análisis del uso de la tierra (6). En ese contexto los resultados obtenidos en este trabajo permiten cuantificar, localmente en el área de estudio, los errores de posición y superficie existentes en los documentos cartográficos elaborados con métodos simples (Sketchmaster) durante el Proyecto Catastro, respecto a la nueva generación de cartografía digital, o Softcopy-Fotogrametría.

Los resultados de la evaluación geométrica de la cartografía elaborada con el Método 1, definidos como de errores de posición espacial de los objetos, se presentan en el cuadro 2 .

El error medio de la posición espacial de los objetos en la cartografía confeccionada con el Método 1 supera los 68 metros de distancia respecto a su posición real en terreno, alcanzando un error probable máximo de 53 metros. Ambos errores a una escala cartográfica 1:50.000 corresponden a 1,36 y $1,07 \mathrm{~mm}$. respectivamente. La magnitud de los errores en las coordenadas $\mathrm{x}$ e y alcanzaron a un error medio de 48 y 47 metros, lo que significa en escala 1: $50.000,0,96$ y $0,95 \mathrm{~mm}$ respectivamente.

Como el área de estudio se ubica en una zona de topografía plana a semiondulada, donde predo-

CUADRO 2

Error de posición de objetos fotointerpretados en la carta IGM-F 101

Position error and coordinates for objects photointerpreted in I.G.M.-F 101.

\begin{tabular}{|lccc|}
\hline \multicolumn{1}{|c}{$\begin{array}{c}\text { Error del Método 1 } \\
\text { (en terreno) }\end{array}$} & $\begin{array}{c}\text { Error de posición } \\
\text { de objetos }(\mathrm{m})\end{array}$ & $\begin{array}{c}\text { I X- error I } \\
(\mathrm{m})\end{array}$ & $\begin{array}{c}\text { I Y- error I } \\
(\mathrm{m})\end{array}$ \\
\hline Error medio & 68,0 & 48,5 & 47,7 \\
Error de muestreo del error medio & 4,3 & 3,2 & 3,1 \\
Error probable & 53,7 & 38,2 & 26,7 \\
& & & \\
\hline \multicolumn{1}{c}{ Error } & Error de posición & I X- error I & I Y- error I \\
de objetos (mm) & $(\mathrm{mm})$ & $(\mathrm{mm})$ \\
(en cartas escala 1:50.000) & 1,36 & 0,96 & 0,95 \\
& 0,08 & 0,06 & 0,06 \\
Error medio & 1,07 & 0,76 & 0,53 \\
Error de muestreo del error medio & & & \\
Error probable & & &
\end{tabular}


BOSQUE 25(1): 87-94, 2004

Cartografía elaborada con un método fotogramétrico simple versus un método digital (Softcopy-Fotogrametría)

mina la categoría de pendiente $0-15 \%$, se puede esperar un error de posición de mayor magnitud en sectores con topografía abrupta como, por ejemplo, la Cordillera de los Andes o Cordillera de la Costa, los cuales pueden superar los 100 metros, considerando las diferencias de relieve existentes y su efecto sobre el desplazamiento radial de los polígonos en la imagen fotográfica (3).

Diferencias de superficie poligonal. Los errores de superficie se determinaron en una submuestra de 8.160 ha, que incluyó 42 polígonos del área de estudio. Los resultados obtenidos se presentan en el cuadro 3 .

La diferencia absoluta de superficie entre ambas cartografías es de 371 ha; esto corresponde a $4,6 \%$ de la superficie total muestreada. Esta diferencia absoluta incluye compensaciones por sobre y subestimación de superficie en los polígonos evaluados. La diferencia real de superficies entre cartografía elaborada con el Softplotter y la cartografía del Proyecto Catastro corresponde 1.078,9 ha (suma de los valores absolutos de sobre y subestimación) sobre un total de 8.160 ha. Esto representa un $13,2 \%$ de la superficie total muestreada.

El error en la delimitacion de una área depende del tamaño, forma y de nitidez de sus límites geográficos $(6,7)$. En el área de estudio la forma de los polígonos es en general irregular, aun cuando los límites de estos son en algunos casos líneas rectas y bien definidas, específicamente en los rodales de plantaciones forestales y áreas urbanas.

En el cuadro 4 se presentan los errores de superficies determinados para la cartografía elaborada en 1994, desagregados según rango de superficies de los polígonos muestreados.

En el cuadro 4 se observa una diferencia absoluta para los tres rangos de superficie de polígonos que supera al 5\% de la superficie muestreada. Por otra parte, la diferencia real de superficies -suma de sobre y subestimaciones- alcanza a un $18,4 \%$ en los polígonos del rango de superficies $100-300$ ha. En las otras clases este es error es inferior al $13 \%$.

Finalmente, se presentan en el cuadro 5 las diferencias de superficies desagregadas por las distintas clases de uso del proyecto Catastro.

Se observa en el cuadro 5 que los mayores errores se producen en las clases de uso cuerpos de agua y agrícola; este error es atingente al proceso de digitalización y no a un error en la fotointerpretación, considerando que en áreas de esas características los límites son de gran nitidez. Los demás usos presentan diferencias absolutas de superficies inferiores entre $10 \%$ y $14 \%$ del área correspondiente a su clase.

\section{CUADRO 3}

Valores máximos, mínimos y total de los 42 polígonos medidos.

Maximum, minimum values and totals for 42 measured polygons.

\begin{tabular}{|l|r|r|r|r|}
\hline \multirow{2}{*}{ Descripción de superficies evaluadas } & \multicolumn{3}{|c|}{ Superficie (ha) } \\
\cline { 2 - 5 } & $\begin{array}{c}\text { Polígono } \\
\text { Medio }\end{array}$ & $\begin{array}{c}\text { Polígono } \\
\text { Mínimo }\end{array}$ & $\begin{array}{c}\text { Polígono } \\
\text { Máximo }\end{array}$ & $\begin{array}{c}\text { Total } \\
\text { Evaluada }\end{array}$ \\
\hline Carta elaborada con Método 1 & 194,5 & 15,3 & $1.637,1$ & $8.160,1$ \\
Carta elaborada con Método 2 & 185,4 & 14,8 & $1.601,0$ & $7.789,4$ \\
Superficie cartográfica común entre Método 1 y 2 & 177,0 & 13,1 & $1.560,6$ & $7.436,1$ \\
Error de sobrestimación Método 1 & 17,4 & 0,4 & 126,5 & 724,0 \\
Error de subestimación de carta Método 1 & 8,4 & 0,0 & 51,4 & 354,9 \\
Diferencia absoluta entre cartas de Métodos 1 y 2 & 9,1 & 0,4 & 40,7 & 371,0 \\
\hline
\end{tabular}


BOSQUE 25(1): 87-94, 2004 Cartografía elaborada con un método fotogramétrico simple versus un método digital (Softcopy-Fotogrametría)

\section{CUADRO 4}

Error de superficie en la cartografía año 1994, según rango de superficies en los polígonos muestreados (ha). Surface error in the 1994 cartography, according to ranking in the tested polygons (ha).

\begin{tabular}{|l|r|r|r|r|}
\hline \multirow{2}{*}{ Descripción de superficies } & \multicolumn{3}{|c|}{ Rango de superficie de los polígonos } \\
& \multicolumn{3}{|c|}{ evaluados (ha) } \\
\cline { 2 - 5 } & $0-100$ & $100-300$ & $>300$ & Total \\
\hline Superficie total muestreada carta elaborada año Método 1 & $1.302,5$ & $2.179,0$ & $4.678,9$ & $8.160,4$ \\
Superficie total muestreada carta elaborada con Método 2 & $1.240,9$ & $2.106,1$ & $4.442,2$ & $7.789,2$ \\
Superficie común entre carta Método 1 y 2 & $1.188,3$ & $1.942,5$ & $4.305,2$ & $7.436,8$ \\
Diferencia absoluta de sup. entre cartas Método 1 y 2 & 61,2 & 72,8 & 236,89 & 371 \\
Diferencia absoluta de sup. en \% de la carta Método 1 & 4,7 & 3,3 & 5,0 & 4,5 \\
Superficie sobrestimada por carta Método 1 & 113,8 & 236,5 & 373,7 & 724,0 \\
Superficie subestimada por la carta Método 1 & 54,4 & 163,6 & 136,8 & 354,9 \\
Diferencia total real de superficies ( sobre + subestimado) & 168,3 & 400,2 & 510,5 & $1.078,9$ \\
Diferencia total real de superficie en \% de la carta Método 1 & 12,9 & 18,4 & 10,9 & 13,2 \\
\hline
\end{tabular}

\section{CUADRO 5}

Error de superficie en la cartografía 1994, según clase de uso actual (ha). Surface error in the 1994 cartography, according to type of current use.

\begin{tabular}{|l|c|c|c|c|c|c|c|}
\hline \multirow{2}{*}{ Superficie evaluada } & \multicolumn{7}{|c|}{ Clases de uso actual del suelo (superficie ha) } \\
\cline { 2 - 7 } & $\begin{array}{c}\text { Urba- } \\
\text { no }\end{array}$ & $\begin{array}{c}\text { Agríco- } \\
\text { la }\end{array}$ & $\begin{array}{c}\text { Prade- } \\
\text { ras }\end{array}$ & $\begin{array}{c}\text { Matorra- } \\
\text { les }\end{array}$ & $\begin{array}{c}\text { Planta- } \\
\text { ciones }\end{array}$ & $\begin{array}{c}\text { Bosque } \\
\text { nativo }\end{array}$ & $\begin{array}{c}\text { Cuerpos } \\
\text { de agua }\end{array}$ \\
\hline $\begin{array}{l}\text { Superficie total muestreada } \\
\text { carta 1994 }\end{array}$ & 75,9 & $1.710,3$ & 479,67 & 374,5 & $5.101,7$ & 41,0 & 377,06 \\
$\begin{array}{l}\text { Superficie total muestreada } \\
\text { carta Softplotter }\end{array}$ & 75,0 & $1.598,0$ & 465,3 & 363,1 & $4.907,1$ & 40,9 & 339,9 \\
$\begin{array}{l}\text { Superficie común entre } \\
\text { cartas 1994-Softplotter }\end{array}$ & 70,6 & $1.533,7$ & 444,07 & 349,3 & $4.718,2$ & 38,0 & 282,2 \\
$\begin{array}{l}\text { Diferencia absoluta de sup. } \\
\text { entre cartas 1994-Softplotter }\end{array}$ & 0,9 & 112,4 & 14,4 & 11,4 & 194,7 & 0,1 & 37,2 \\
$\begin{array}{l}\text { Diferencia absoluta en \% } \\
\text { de carta 1994 }\end{array}$ & 1,2 & 6,6 & 3,0 & 3,0 & 3,8 & 0,2 & 9,9 \\
$\begin{array}{l}\text { Superficie sobrestimada } \\
\text { de carta 1994 }\end{array}$ & 5,4 & 176,6 & 35,6 & 25,1 & 383,5 & 3,0 & 94,85 \\
$\begin{array}{l}\text { Superficie subestimada } \\
\text { de carta 1994 }\end{array}$ & 4,4 & 64,2 & 21,23 & 13,9 & 190,6 & 2,9 & 57,67 \\
$\begin{array}{l}\text { Diferencia total real de } \\
\text { superficies. Suma de } \\
\text { sobre y subestimaciones }\end{array}$ & 9,8 & 240,83 & 56,8 & 39,0 & 574,08 & 5,9 & 152,52 \\
$\begin{array}{l}\text { Diferencia real de superficie } \\
\text { en \% de la carta 1994 }\end{array}$ & 12,9 & 14,1 & 11,8 & 10,4 & 11,3 & 14,5 & 40,4 \\
\hline
\end{tabular}


BOSQUE 25(1): 87-94, 2004

Cartografía elaborada con un método fotogramétrico simple versus un método digital (Softcopy-Fotogrametría)

\section{CONCLUSIONES}

De la información antes expuesta se concluye que el error de localización de los objetos, referido a las coordenadas $x$ e $y$, es superior a $50 \mathrm{~m}$ en la carta elaborada con el Método 1. Estos errores generan una diferencia de posición espacial en los límites de los polígonos, que se sitúa en el rango de 50 a $60 \mathrm{~m}$ en el área estudiada. Aun cuando esta comparación es de carácter local y se refiere a una superficie y de prueba reducida, se pueden esperar tendencias y errores mayores en áreas con topografía más abrupta como, por ejemplo, en las Regiones X y XI.

La magnitud de los errores de superficie encontrados en el área estudiada es inferior al 5\% en relación a la cartografía elaborada con el Método 2. El error real, incluyendo sub y sobre estimaciones; sin embargo; supera el $12 \%$. Aun cuando este valor está en el margen superior de lo aceptado para estudios regionales $(3,6,8)$, es aconsejable considerar en próximas actualizaciones cartográficas del país correcciones geométricas, con el objeto de mejorar las características proyectivas de la valiosa información alfanumérica generada por el Proyecto Catastro. Estas correcciones deberán disminuir los errores referenciales a valores inferiores al $1 \%(2)$.

\section{BILIOGRAFIA}

(1) CONAF. 1999. Catastro y evaluación de recursos vegetacionales de Chile. Informe Nacional, marzo 1999, $85 \mathrm{p}$.

(2) SANDOVAL, V., P. REAL. Establecimiento de un Sistema de Seguimiento y Monitoreo del estado de conservación de las formaciones vegetales y naturales de Chile. Serie Técnica. Universidad Austral de Chile, 2001. Valdivia. $41 \mathrm{p}$.

(3) SANDOVAL, V. Evaluación de tres métodos de transferencia CARTA-FOTO. Tesis de Grado, Facultad de Ciencias Forestales, Universidad Austral de Chile, Valdivia. 1982. $45 \mathrm{p}$.

(4) HILDEBRANDT, G. Fernerkundung und Luftbildmessung. Herbert Wichmann Verlag, Heidelberg Deutschland 1996. $676 \mathrm{p}$.

(5) PRÖBSTING T. Luftbildgetutzte Vefahren für die Forstwirtschaft. Bedarf, Anwendungbereiche, Auswertungssyteme. Cuvillier Velag Göttingen. 1997. 175 p.

(6) AKCA A., J. SABOROWSKY. Überprufung von Vegetationskartierung aus großmaßstäbigen Luftbilder und Entwiklung eines Prüfkonzeptes. Projectbericht. Institut für Forsteinrichtung und Ertragskunde, Georg-AugustUniversität Göttingen, 1998. $41 \mathrm{p}$

(7) AKCA, A., SCHNOKLAKE. Luftbild basierte Waldzustandserffassung auf Landkreisebene im Privatwald von Schleswig-Holstein. Inst. f. Forseinricht. und Ertragskunde U. Göttingen. Dezember 1998. 65 p.

(8) REAL, P., V. SANDOVAL. 1999. Sistema de consultas alfanumérica y cartográfico del Catastro Forestal Chileno, Seminario Silvo, Concepción, Chile. 1999. 20 p. 